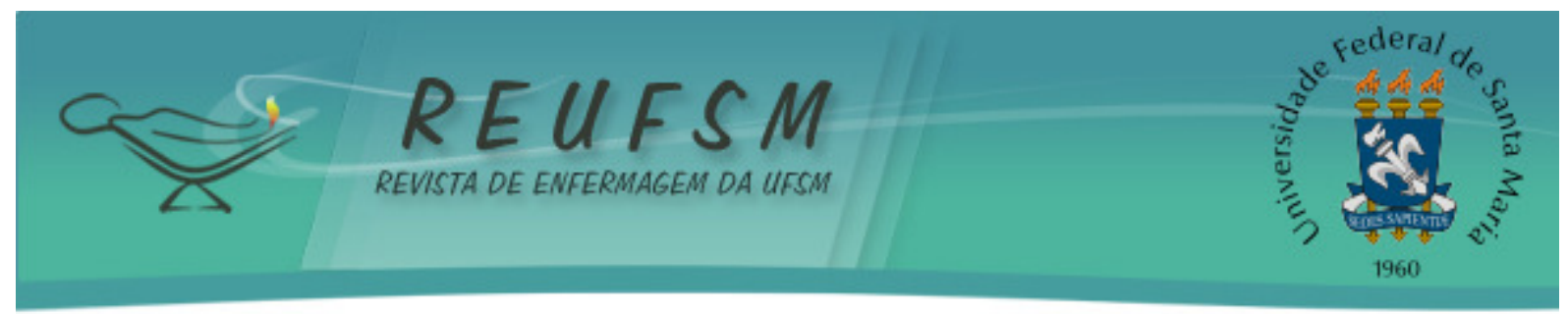

ARTIGO ORIGINAL

\title{
INJEÇÕES INTRAMUSCULARES VENTRO-GLÚTEA E A UTILIZAÇÃO PELOS PROFISSIONAIS DE ENFERMAGEM
}

\section{VENTRO-GLUTEAL INTRAMUSCULAR INJECTIONS AND UTILIZATION BY PROFESSIONAL NURSING}

\section{INYECCIONES INTRAMUSCULARES VENTROGLÚTEA Y LA UTILIZACIÓN POR LOS PROFESIONALES DE ENFERMERÍA}

\author{
Indiara Sartori Dalmolin ${ }^{1}$ \\ Vera Lucia Freitag ${ }^{2}$ \\ Sidnei Petroni ${ }^{3}$ \\ Marcio Rossato Badke ${ }^{4}$
}

RESUMO: Objetivo: investigar os índices de utilização da região ventro-glútea para administração intramuscular pelos profissionais de enfermagem. Método: pesquisa quantitativa descritiva, com participação de 68 sujeitos. Para a coleta dos dados aplicou-se um questionário, sendo a análise processada através de estatística descritiva, com o Software SPSS 15.0 e o Excel. A pesquisa teve aprovação do Comitê de Ética em Pesquisa da Universidade Federal de Santa Maria sob o número 0285.0.243.000-11 em 03/01/2012. Resultados: os dados revelaram que $5,9 \%$ dos profissionais utilizam às vezes a região ventro-glútea, mas há uma preferência pela região dorso-glútea para a administração de fármacos intramusculares nas três instituições investigadas. Conclusão: a aplicação de injeções na região ventro-glútea foi pouco utilizada pelos profissionais pesquisados e observou-se falta de conhecimento dos profissionais sobre a técnica correta de aplicação.

Descritores: Enfermagem; Injeções intramusculares; Educação em saúde.

ABSTRACT: Aim: to investigate the utilization rates of the ventro-gluteal region for intramuscular by nursing professionals. Method: quantitative and descriptive research, with participation of 68 subjects. To collect the data, was applied a questionnaire being processed through the analysis of descriptive statistics with the software SPSS 15.0 and Excel. The research was approved by the Ethics Committee at the Santa Maria Federal University with number 0285.0.243.000-11 on 03/01/2012. Results: data revealed that $5.9 \%$ of the professionals sometimes use the ventro-gluteal region, but there is a preference for back-buttocks region for the intramuscular administration of drugs in the three institutions investigated. Conclusion: the application of injections in ventralgluteal region was little used by the professionals surveyed and observed lack of professional knowledge on correct technique of application.

Descriptors: Nursing; Injections, intramuscular; Health education.

\footnotetext{
${ }^{1}$ Acadêmica do $8^{\circ}$ semestre do curso de Graduação em Enfermagem da Universidade Federal de Santa Maria (UFSM), Campus Palmeira das Missões, RS, Brasil. Bolsista do Programa REUNI de Bolsas de Iniciação Científica. E-mail: indiarasartoridalmolin@yahoo.com.br

${ }^{2}$ Acadêmica do $8^{\circ}$ semestre do curso de Graduação em Enfermagem da Universidade Federal de Santa Maria (UFSM), Campus Palmeira das Missões, RS, Brasil. Bolsista do Fundo de Incentivo à Extensão (FIEX). E-mail: verafreitag@hotmail.com

${ }^{3}$ Biólogo (UFSM), Doutorado em Ciências Biológicas (Anatomia) pela Universidade Estadual Paulista Júlio de Mesquita Filho. Professor Adjunto II da UFSM, Campus Palmeira das Missões, RS, Brasil. E-mail: sidneipetroni@yahoo.com.br

${ }^{4}$ Enfermeiro, Mestre em Enfermagem (UFSM), Doutorando do Programa de Pós Graduação em Enfermagem (PPGEnf) da Universidade Federal de Pelotas (UFPel). Professor Assistente II da UFSM, Campus Palmeira das Missões, RS, Brasil. E-mail: marciobadke@yahoo.com.br
} 


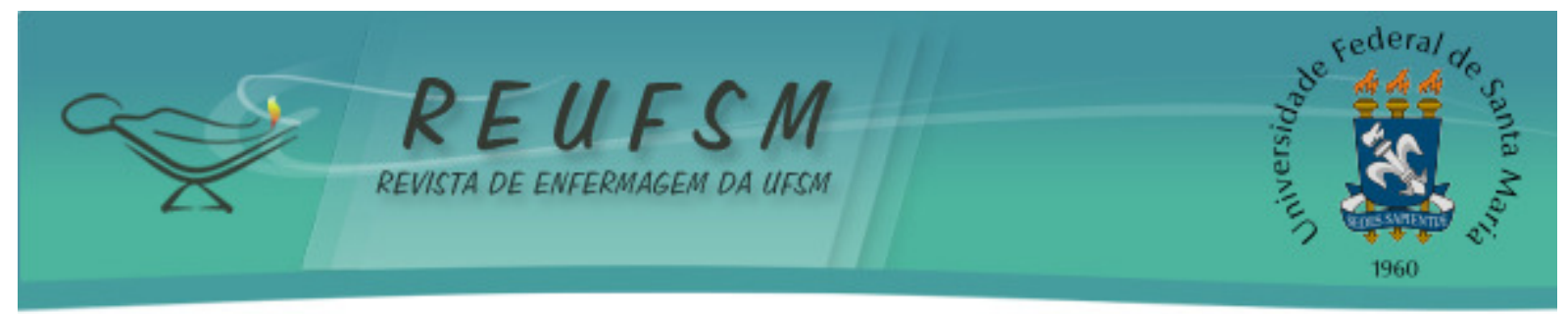

RESUMEN: Objetivo: investigar las tasas de utilización de la región ventroglútea por vía intramuscular de los profesionales de enfermería. Método: investigación cuantitativa descriptiva, con participación de 68 sujetos. Para la recolección de los datos se aplicó un cuestionario y análisis se procesó a través de la estadística descriptiva con el Software SPSS 15.0 y Excel. La investigación fue aprobada por el Comité de Ética de la Universidade Federal de Santa María, con el número 0285.0.243.000-11 en 03/01/2012. Resultados: los datos revelaron que el $5,9 \%$ de los profesionales utilizan a veces la región ventroglútea, pero existe una preferencia para la región dorsoglútea para la administración intramuscular de fármacos en las tres instituciones investigadas. Conclusión: la aplicación de inyecciones en la región ventroglútea fue poco utilizado por los profesionales encuestados y observó la falta de conocimientos de los acerca de la técnica correcta de aplicación.

Descriptores: Enfermería; Inyecciones intramusculares; Educación en salud.

\section{INTRODUÇÃO}

A administração de fármacos por via intramuscular (IM) é um procedimento utilizado regularmente na prática clínica da Enfermagem. ${ }^{1}$ A região ventro-glútea (VG) é a mais indicada em relação às demais, por ser facilmente delimitada pelos acidentes anatômicos palpáveis utilizados na técnica de Hochstetter. Esta técnica foi proposta pelo anatomista suíço Von Hochstetter e seus colaboradores em 1954, que realizaram uma profunda investigação anatômica da região VG, com o objetivo de explicar as várias intercorrências da aplicação intraglútea. ${ }^{2}$

A administração de um medicamento por via IM abrange mais do que a injeção de um fármaco, requer também, uma avaliação da região e do músculo mais apropriados. Este procedimento deve ser realizado por profissionais que conhecem os aspectos fundamentais para a execução técnica, ou seja, equipe de enfermagem. Apesar de ser considerado por muitos como um procedimento simples, a prática e os dados científicos apresentam inúmeras intercorrências relacionadas à aplicação de medicamentos por via $\mathrm{IM}^{3}$ Cabe ressaltar que, na busca por alternativas para eliminar as complicações relacionadas ao uso do músculo glúteo máximo, na aplicação IM, Von Hochstetter identificou e comprovou, por meio de consistentes investigações, que a região VG é o local que menor risco oferece ao paciente. ${ }^{4}$ Este autor acrescenta que relatos de lesões de necrose tecidual, contraturas musculares, fibrose e até perda de movimentos articulares foram observado em pessoas que fizeram uso de medicação intramuscular, ocorrências comuns quando da utilização dos músculos deltóide, glúteo máximo e vasto lateral da coxa, que podem ser traduzidos como os eleitos para aplicação de medicações IM na prática de enfermagem. ${ }^{4}$

O plano muscular da região VG é constituído pelos músculos glúteo médio e mínimo que ficam cobertos pelo músculo glúteo máximo. É considerado pela literatura o local mais seguro e adequado para a aplicação IM em adultos, criança e bebês. ${ }^{5}$ Esta região é a mais indicada devido às suas peculiaridades: maior espessura dos ventres musculares (músculos glúteo médio e mínimo), local livre de vasos e nervos importantes tanto em adultos como em crianças e menor espessura de tecido subcutâneo se comparada às outras regiões de aplicação, além do fácil acesso tanto em decúbito ventral, dorsal ou lateral. A direção das fibras musculares previne o deslizamento do material injetado para a região do nervo isquiático (ciático), livrando-o de irritações. Acrescenta-se também a vantagem de que a epiderme nesta região tem menor concentração de germes patogênicos anaeróbios quando comparada a dorso-glútea (DG), pois é menos passível de ser contaminada com fezes e urina, principalmente em pacientes acamados. ${ }^{2}$

Indiscutivelmente, a região VG é a mais segura, entretanto é minimamente utilizada, como foi identificado em estudos realizados no estado de São Paulo, Brasil., ${ }^{2,6-7}$ 


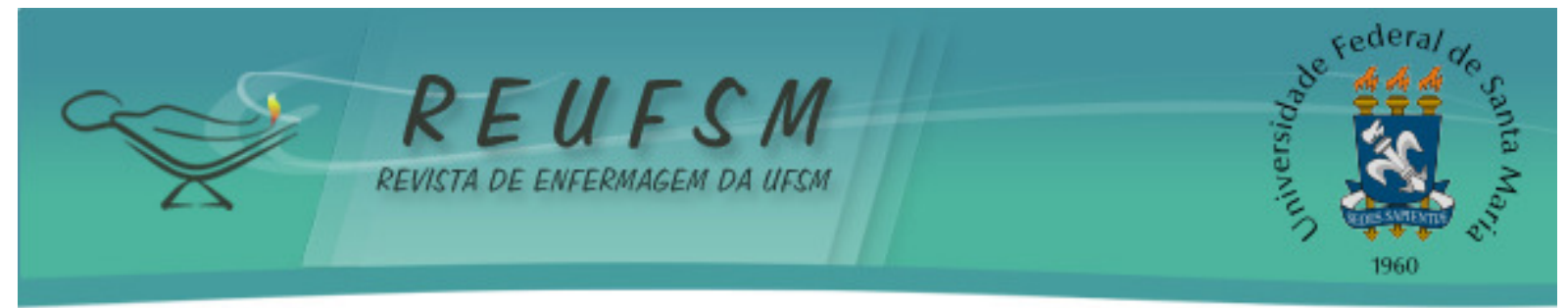

Outra pesquisa, efetivada em uma província canadense, também confirmou que os enfermeiros não utilizam preferencialmente a região VG para a administração de injeções IM em adultos, como recomendado pela literatura. ${ }^{8}$ Uma das dificuldades se pauta na crença de que na região VG as pessoas apresentam maior sensibilidade dolorosa, acrescese ainda o fato da preferência por parte da maioria dos profissionais da área da enfermagem resistir a mudanças devido a sua insegurança ou pelo apego às técnicas tradicionais, utilizando principalmente a região DG como o local eleito.

Em meio às lacunas teóricas e práticas nos hospitais de ensino da região de abrangência da Universidade Federal de Santa Maria (UFSM), Campus de Palmeira das Missões/RS, discentes e docentes envolvidos no trabalho de pesquisa observaram empiricamente, pouca ou nenhuma utilização da região VG para a aplicação medicamentosa.

Diante da realidade exposta, esta investigação teve como foco investigar os índices de utilização da região VG para administração IM pelos profissionais de enfermagem. Para melhor sustentar a pesquisa partiu-se da perspectiva de responder à seguinte pergunta: qual o índice de aplicabilidade de injeção IM na região VG pelos profissionais da equipe de enfermagem?

\section{MÉTODO}

Trata-se de uma pesquisa de caráter quantitativo descritivo. A pesquisa quantitativa refere-se ao plano geral do pesquisador para responder às questões de pesquisa ou testar as suas hipóteses. Em um estudo quantitativo, o delineamento de pesquisa apresenta as estratégias que o pesquisador planeja adotar para desenvolver informações precisas e interpretáveis. ${ }^{9}$

Além disso, a pesquisa quantitativa considera que tudo pode ser quantificável, o que significa traduzir em números, opiniões e informações para classificá-las e analisá-las. Requer o uso de recursos e de técnicas estatísticas (percentagem, média, moda, mediana, desvio-padrão, coeficiente de correlação, análise de regressão, entre outras). ${ }^{10}$

Esta investigação foi desenvolvida junto a três hospitais de pequeno e médio porte da região norte do estado do RS, tendo como participantes os profissionais da equipe de enfermagem (enfermeiros, técnicos e auxiliares de enfermagem) que aceitaram voluntariamente participar do estudo, estando presente/trabalhando nos dias de coleta e assinando o Termo de Consentimento Livre e Esclarecido (TCLE). Os sujeitos foram convidados a participar da pesquisa com antecedência, sendo agendados dias e horários para a aplicação dos questionários. E a definição dos participantes deu-se pelo sistema de amostra aleatória simples. ${ }^{9}$

0 instrumento utilizado para a coleta dos dados foi um questionário elaborado para este estudo, composto por seis questões sociodemográficas, cinco questões fechadas e três abertas que abrangeram os conhecimentos sobre a anatomia da região glútea e VG, as técnicas utilizadas nas aplicações intramusculares e a especificidade da região VG. A análise das informações quantitativas foi processada por meio de estatística descritiva, com a utilização do Software SPSS (Statistical Package for the Social Sciences) 15.0 e do Excel.

Para valorizar e assegurar os princípios éticos durante o processo da pesquisa, o projeto foi registrado no Sistema de Informações para o Ensino (SIE) da Universidade Federal de Santa Maria, sob o número 030333. Os dados foram coletados após a aprovação do Comitê de Ética em Pesquisa da UFSM com protocolo CAAE (Certificado de Apresentação para Apreciação Ética) número CAAE 0285.0.243.000-11 em 03/01/2012. Ademais, apoiouse nas orientações e disposições da Resolução n 196/96, do Ministério da Saúde, que descrevem as diretrizes e normas que regulamentam os processos de investigação envolvendo seres humanos, no Brasil. ${ }^{11}$ 


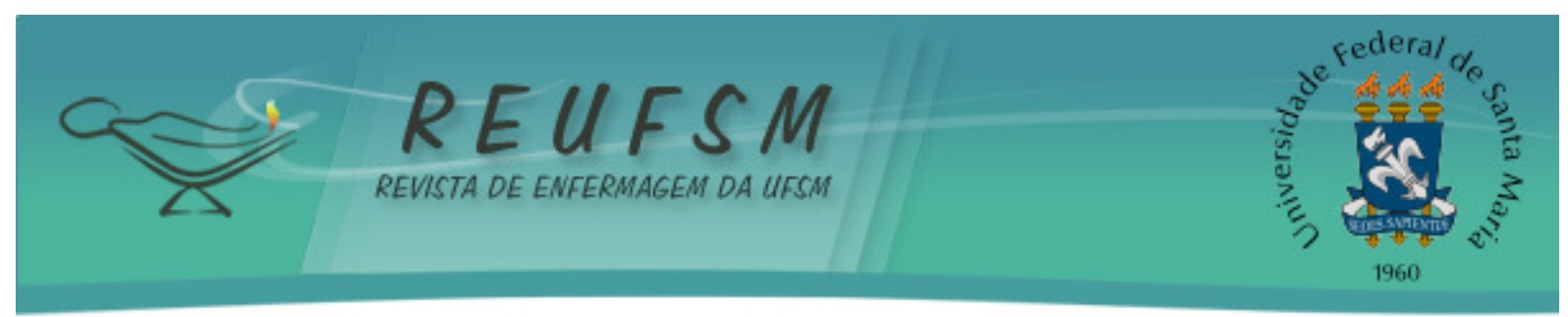

\section{RESULTADOS E DISCUSSÃO}

\section{Caracterização dos sujeitos}

Participaram do estudo 68 profissionais da área da enfermagem, sendo seis $(8,8 \%)$ enfermeiros, 41 (60,3\%) técnicos de enfermagem e 21 (30,9\%) auxiliares de enfermagem. Destes 68 sujeitos, $60(88,2 \%)$ são do sexo feminino e oito $(11,8 \%)$ são do sexo masculino.

Considerando $100 \%$ da amostra pesquisada, $41(60,3 \%)$ pertencem ao Hospital A (de médio porte), 15 (22,1\%) ao Hospital B e 12 (17,6\%) ao Hospital C (sendo estes últimos de pequeno porte).

A amplitude de idade dos indivíduos inclusos na pesquisa ficou entre 21 e 57 anos e a amplitude do tempo de trabalho na instituição ficou entre dois meses e 34 anos.

\section{Entre a teoria e a prática na abordagem ventro-glútea pelos profissionais de enfermagem}

Entre os sujeitos questionados sobre o conhecimento do músculo tensor da fáscia lata, 20 (29,4\%) responderam que conhecem este músculo, 46 (67,6\%) responderam que não o conhecem e dois (3\%) não responderam a esta indagação. Com relação ao músculo glúteo médio e glúteo mínimo $38(55,9 \%)$ responderam que conhecem este músculo e $30(44,1 \%)$ não o conhecem.

Quando interrogados sobre o conhecimento na aplicação de injeção intramuscular na região VG, com a utilização da técnica de Hochstetter, $22(32,4 \%)$ responderam que conhecem a técnica, $45(66,2 \%)$ não a conhecem e um $(1,4 \%)$ não respondeu a esta pergunta.

Em relação à aplicação de injeção intramuscular na região VG, quatro $(5,9 \%)$ profissionais costumam aplicar neste local, $39(57,3 \%)$ não usam esta técnica e $25(36,8 \%)$ não responderam a este questionamento.

Nesta perspectiva, a análise quantitativa identificou que os profissionais de enfermagem pesquisados, em geral, não utilizam a região VG para a aplicação de injeções/medicações e 67,16\% relataram desconhecimento referente à sua delimitação geométrica. Dados de outro estudo corroboram com os resultados desta pesquisa, pois mostram que a administração IM na região VG é uma novidade para os indivíduos pesquisados em seu trabalho, e que, portanto, desconhecem a real importância desse local para a administração de medicamentos. ${ }^{3}$

Percebe-se assim a existência de outras regiões de maior aceitação pelas equipes de Enfermagem no momento da escolha em administrar medicações IM. Além disso, durante a análise dos dados, observou-se que, apesar de 32,4\% demonstraram conhecer a técnica de Hochstetter, apenas 5,9\% destes profissionais utilizam esse local em sua vida profissional. Assim, cabe refletir o porquê dos profissionais que conhecem a técnica não a utilizarem, levando em consideração os seguintes fatores: o medo, o aspecto cultural dos pacientes que não aceitam esta técnica, a resistência às mudanças no ambiente de trabalho, entre outros. Logo, é relevante explorar essa questão num estudo futuro, a fim de conhecer os reais motivos da não utilização desta região anatômica para a aplicação de medicamentos por via IM.

Outro estudo revelou que os enfermeiros na prática, continuam utilizando e treinando os estudantes de enfermagem para a aplicação de injeção IM na região DG como o local de escolha, apesar das inúmeras evidências sobre as complicações associadas ao uso deste local. Tornando-se a adesão a região VG um desafio, sobretudo, devido à falta de enfermeiros qualificados para a manipulação desta região anatômica. ${ }^{12}$

Baseando-se nisso, entende-se que a mudança dessa realidade depende da formação acadêmica e da atuação da equipe de enfermagem, que ao receber treinamento técnico adequado, passe a incorporar tal procedimento em sua prática. O Ministério da Saúde confirma a expectativa de que o enfermeiro deve exercer o seu papel de educador junto à comunidade a que 


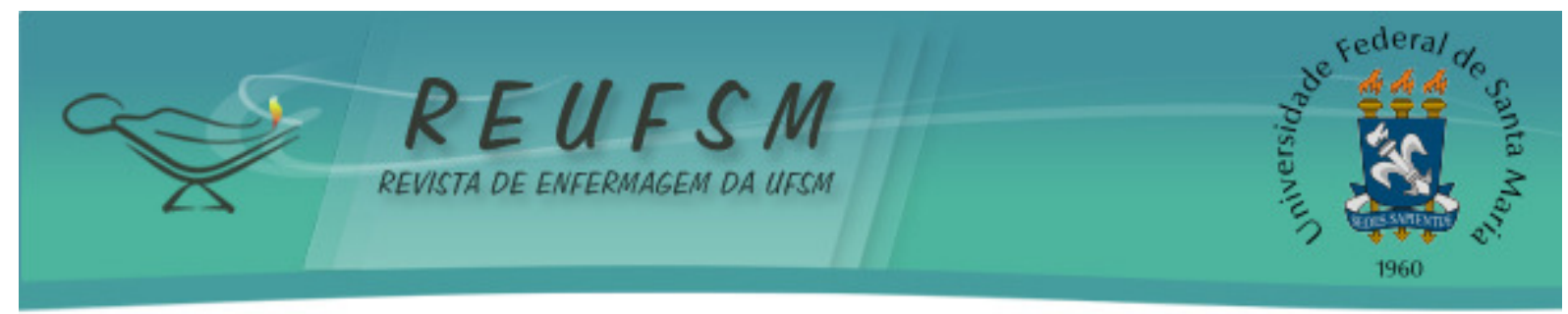

pertence através de um processo de educação permanente em saúde, ${ }^{13}$ com a equipe profissional e com os usuários do serviço. Porquanto que, as mudanças precisam ser agregadas ao processo de trabalho, na busca por melhores técnicas e condições para a assistência prestada.

Por conseguinte, a literatura afirma que os medicamentos administrados erroneamente podem causar prejuízos/danos ao paciente devido a fatores como incompatibilidade farmacológica, reações indesejadas e interações. ${ }^{6}$ Outras possíveis complicações são: a formação de abscesso, eritema, infiltrações no tecido subcutâneo, embolias, lesões nervosas, dor aguda e diminuição da sensibilidade. Destaca-se que o grupo das crianças é o que apresenta mais complicações e onde há várias descrições na literatura de reações encontradas após aplicações múltiplas de antibióticos neste grupo populacional. ${ }^{6}$

Logo, é necessário que o profissional que administra medicamentos esteja consciente e seguro de sua ação e possua conhecimentos ou acesso às informações necessárias. Tais aspectos evidenciam a necessidade de supervisão das atividades de enfermagem, pelos enfermeiros, durante o preparo e administração de medicamentos, já que esse é o único profissional da equipe de enfermagem que deveria contar, na sua formação, com conhecimentos suficientes para conduzir tal procedimento de modo seguro.

Nesse contexto, as regiões recomendadas para a aplicação IM devem ser, salvo algumas exceções, as da seguinte sequência: região VG, região DG, região da face anterolateral da coxa (FALC) e região deltóidea (D). ${ }^{2}$ Contudo, durante a coleta dos dados, quando pediu-se para que descrevessem a técnica de aplicação VG, apenas três dos 68 sujeitos a descreveram de forma correta, revelando precário conhecimento da equipe de enfermagem sobre o tema, pois mesmo entre os que afirmaram conhecer a técnica, a maioria não soube descrevê-la corretamente. Este pode ser mais um motivo da subutilização desta região de aplicação $I M$, as dúvidas em relação à delimitação geométrica. Assim, ressalta-se que a busca por profissionais cada vez mais qualificados conduz a maiores investimentos em capacitação dos trabalhadores da saúde.

Logo, é importante definir um modelo de política de formação, capacitação e educação em saúde para os recursos humanos. As capacitações na área da saúde devem ser consideradas estratégicas, podendo constituir-se num espaço concreto de construção de competências técnicas, política, e ética para o fortalecimento dos recursos humanos. ${ }^{14}$ Dessa forma, torna-se fundamental citar a Política Nacional de Educação Permanente em Saúde (EPS), criada pelo Ministério da Saúde em 2004, que propõe que a transformação das práticas profissionais devem estar baseadas na reflexão crítica, em espaços coletivos, em rodas de discussão, a partir da problematização da realidade do trabalho, identificando as necessidades de capacitação. ${ }^{13}$

A edificação de propostas de educação em saúde necessita estar articulada com as demandas dos sujeitos, sejam eles trabalhadores e/ou usuários. Através desta organização as ações educativas tornam-se profícuas e impactam transformações no processo de trabalho. A EPS deve constituir o pensar e o agir da equipe, com a finalidade de produzir interação entre os profissionais, crescimento pessoal e profissional de toda a equipe, estimulando melhorias no atendimento e no trabalho em equipe. ${ }^{15}$

A despeito da recomendação expressa na literatura técnica da área, a prática atual da enfermagem, com base nos dados desta investigação, indica que a administração de injeção IM segue a seguinte ordem de preferência: região DG, região $D$, região da FALC e por último, a região VG. Corroborando com isso, um estudo que propôs um modelo de delimitação geométrica para injeções VG, faz referência a um hospital escola do interior do Estado de São Paulo, no tocante a região utilizada com maior frequência para aplicação de injeções por via IM, entre integrantes da equipe de Enfermagem, demonstrando considerável inversão na sequência prioritária mais adequada a partir dos referenciais teóricos: região DG com 65,62\%, D 31,25\%, FALC 15,62\% e VG 12,5\%.² 


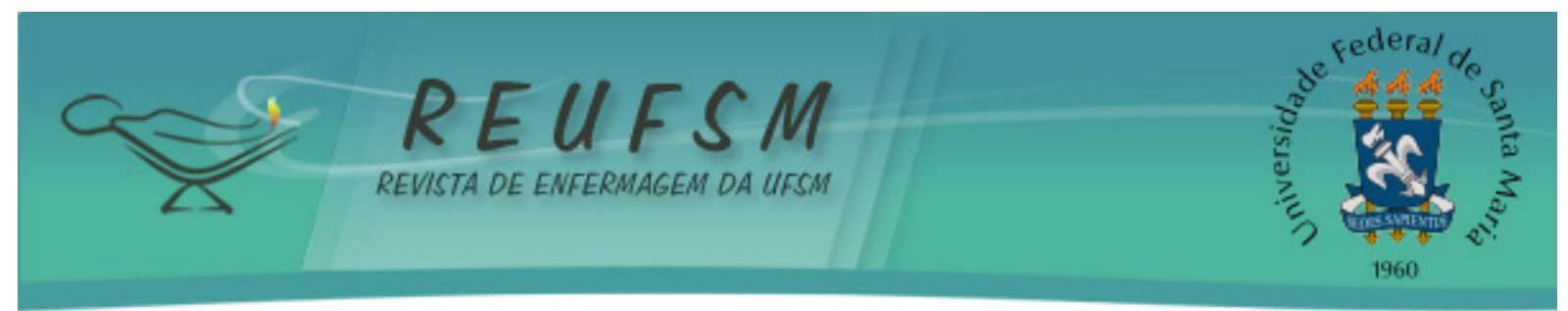

Dessa forma, os resultados obtidos no presente trabalho e que focou três Instituições Hospitalares da região norte do estado do RS, com abrangência da UFSM, confirmam os resultados obtidos por investigações realizadas na região sudeste do Brasil e no Canadá. ${ }^{2,4,6-8}$ Isto mostra o desconhecimento da técnica de injeções VG e sua aplicabilidade, que embora indicada como de primeira escolha, é pouco utilizada. Além disso, outra pesquisa revelou que no Reino Unido e nos Estados Unidos da América os enfermeiros também utilizam com maior frequência a região DG ao invés da VG, o que evidencia que esta problemática não afeta apenas a sociedade brasileira. ${ }^{1}$

De tal forma buscou-se com este artigo refletir sobre o distanciamento entre a teoria e a prática na abordagem IM de medicações VG existente na prática clínica dos profissionais de enfermagem e também na sociedade. Os resultados evidenciaram a necessidade de se reforçar esta temática nos currículos universitários e nos hospitais de ensino, a fim de que se apliquem os conhecimentos teóricos que se tem produzido sobre o tema na formação de novos profissionais, para que os mesmos possam estar bem preparados tecnicamente e não reproduzam a cisão constatada nesta pesquisa em relação ao binômio teoria/prática.

\section{CONCLUSÃO}

Os resultados deste estudo permitiram concluir que a região VG ainda é pouco conhecida e minimamente utilizada; os profissionais mostraram baixo conhecimento sobre esta região e apego a locais tradicionais, como as regiões D e DG para a aplicação de IM; de acordo com os sujeitos pesquisados há ausência de critérios para a seleção da região mais segura para procedimentos intramusculares, à medida que a região DG é utilizada como primeira escolha.

Destaca-se que a amostra não muito numerosa de sujeitos, a qual ficou restrita ao contingente populacional de três hospitais, pode ser um fator limitante da pesquisa. Contudo, dentro dos contextos destas instituições os dados impulsionaram a reflexão sobre a prática da enfermagem, em duas perspectivas: na visão docente e na visão discente. Em relação à primeira, é importante ressaltar o papel das Instituições de Ensino Superior (IES) e Técnico, na forma como abordam as disciplinas de fundamentos de enfermagem. E em relação à segunda, permite aos estudantes pensar, refletir e atuar no contexto da enfermagem em campos de estágios, como um período que antecede a vida profissional, logo, devem se habituar a aproximar a prática ao máximo da teoria, para que, na futura vida profissional possam desenvolver técnicas seguras, eficazes e com maior resolubilidade para o paciente e para a profissão.

Sugere-se trabalhar conceitos teóricos e práticos em relação a esta região anatômica nos conteúdos básicos e específicos do curso de graduação em Enfermagem, estimulando os futuros profissionais a aumentarem a utilização e aplicabilidade por esta via em seus campos de trabalho.

\section{REFERÊNCIAS}

1. Donaldson C, Green J. Using the ventrogluteal site for intramuscular injections. Nurs Times 2005; 101(16):36-8.

2. Meneses AS, Marques IR. Proposta de um modelo de delimitação geométrica para a injeção ventro-glútea. Rev bras enferm [internet]. 2007 [acesso 2012 jun 29];60(5):552-8. Disponível em: http: / /www.scielo.br/scielo.php?script=sci_arttext\&pid=S0034-71672007000500013

3. Godoy S, Nogueira MS, Mendes IA. Aplicação de medicamentos por via intramuscular: análise do conhecimento entre profissionais de enfermagem. Rev Esc Enferm USP [internet]. 2004 [acesso 2012 jun 17];38(2):135-142. Disponível em: http://www.scielo.br/pdf/reeusp/v38n2/03.pdf 


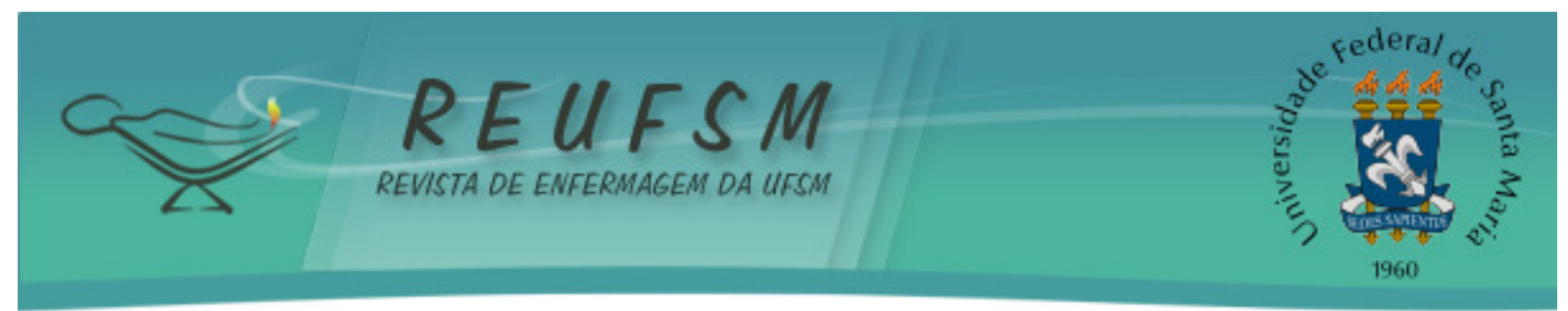

4. Castellanos BEP. Região ventro-glútea: local seguro para aplicação de injeção por via intramuscular. São Paulo, Escola de Enfermagem da USP; 1975.

5. Cook IF, Murtagh J. Ventrogluteal area - a suitable site for intramuscular vaccination for infants and toddlers. Vaccine 2006;24(13):2403-8.

6. Silva DO, Grou CR, Miasso Al, Cassiani SHDB. Preparo e administração de medicamentos: análise de questionamentos e informações da equipe de enfermagem. Rev latinoam enferm [internet]. 2007 [acesso 2012 jun 17];15(5):1010-1017. Disponível em: http://www.scielo.br/pdf/rlae/v15n5/pt_v15n5a19

7. Potter PA, Perry AG. Fundamentos de enfermagem. $5^{\text {th }}$ ed. Rio de Janeiro: Elsevier; 2004.

8. Walsh L, Brophy K. Staff Nurses sites of choice for administering injections to adult patients in the acute care setting. Journal Adv Nurs 2011;67(5):1034-40.

9. Polit DF, Beck CT, Hungler BP. Fundamentos de Pesquisa em Enfermagem: métodos, avaliação e utilização. $5^{\text {th }}$ ed. Porto Alegre: Artmed; 2004.

10. Moresi E. Metodologia da Pesquisa. Programa de Pós-Graduação Stricto Sensu em Gestão do Conhecimento e Tecnologia da Informação [internet]. 2003 [acesso 2012 jul 10]. Disponível em:http://www.unisc.br/portal/upload/com_arquivo/metodologia_da_pesquisa..pdf

11. Brasil, Ministério da Saúde. Conselho Nacional de saúde. Resolução 196/96 sobre pesquisa envolvendo seres humanos. Brasília: Diário Oficial da União; 1996 [acesso 2012 jun 17]. Disponível em: http://bvsms.saude.gov.br/bvs/publicacoes/normas_pesquisa_sereshumanos.pdf

12. Cocoman A, Murray J. Recognizing the evidence and changing practice on injections sites. Br J Nurs 2010;19(18):1170-4.

13. Brasil, Ministério da Saúde. Política de Educação e Desenvolvimento para o SUS: Caminhos para a Educação Permanente em Saúde. Polos de Educação Permanente em Saúde. Brasília: Ministério da Saúde; 1 ed. 2004 [acesso 2012 jul 20]. Disponível em:: http://bvsms.saude.gov.br/bvs/publicacoes/politica2_vpdf.pdf.

14. Melo MLC, Nascimento MAA. Treinamento Introdutório para Enfermeiras Dirigentes: possibilidades para gestão do SUS. Rev bras enferm [internet]. 2003 [acesso 2012 jun 17];56(6):674-677. Disponível em: http://www.scielo.br/scielo.php?pid=S003471672003000600017\&script=sci_arttext

15. Silva LAA da, Bonacina DM, Andrade A de, Oliveira TC de. Desafios na construção de um projeto de educação permanente em saúde. Rev enferm UFSM [internet]. 2012 [acesso 2013 mar 19]; 2(3):496-506. Disponível em: http://cascavel.ufsm.br/revistas/ojs2.2/index.php/reufsm/article/view/5364/pdf

Data de recebimento: 08/03/2013

Data de aceite: $11 / 09 / 2013$

Contato com autor responsável: Indiara Sartori Dalmolin

E-mail: indiarasartoridalmolin@yahoo.com.br

Endereço: Linha Dalmolin, Interior, Caiçara (RS), Brasil, CEP: 98440-000 